\title{
The Genomic Dynamics and Evolutionary Mechanism of the Pi2/9 Locus in Rice
}

\author{
Bo Zhou, ${ }^{1}$ Maureen Dolan, ${ }^{2}$ Hajime Sakai, ${ }^{2}$ and Guo-Liang Wang ${ }^{1}$ \\ ${ }^{1}$ Department of Plant Pathology, the Ohio State University, Columbus OH 43210, U.S.A.; ${ }^{2}$ DuPont Crop Genetics, \\ Experimental Station, Wilmington, DE 19880, U.S.A.
}

Submitted 6 June 2006. Accepted 26 July 2006.

\begin{abstract}
The Pi2/9 locus contains at least four resistance specificities to Magnaporthe grisea and belongs to a gene complex comprised of multiple genes that encode highly homologous nucleotide binding site (NBS) and leucine rich repeat (LRR) proteins. To investigate the genetic events involved in the evolution of the Pi2/9 locus, we analyzed the Pi2/9 locus at the inter- and intralocus levels in five rice cultivars. The NBS-LRR genes in the five cultivars belong to the same phylogenetic clade among rice NBS-LRR genes, and all have a phase- 2 intron at the $\mathrm{N}$-terminus. However, the paralogs within each haplotype show a significant sequence divergence and their N-terminal intron and $5^{\prime}$ regulatory regions are very different. On the contrary, the orthologs from different haplotypes are highly similar, indicating an obvious orthologous relationship has been maintained during the evolution of the Pi2/9 locus. These results suggest that sequence diversification in the $5^{\prime}$ regulatory regions and N-terminal introns of the paralogs may have led to suppression of meiotic recombination between the paralogs within each haplotype, facilitating the maintenance of the orthologous relationship among rice cultivars. Our observations provide valuable insight into the genomic dynamics and evolutionary mechanism of an NBS-LRR resistancegene complex in rice.
\end{abstract}

Plants perceive signals from pathogens during infection. One of the common features in response to pathogen infection is the hypersensitive response (HR) that leads to cell death at the site of infection. The majority of plant resistance $(R)$ genes trigger HR when the plants are invaded by a strain carrying the corresponding avirulence gene. The genes coding for proteins with a nucleotide-binding site (NBS) and leucine-rich repeat (LRR) domain represent the largest plant $R$-gene family, which is further classified into two categories depending on whether

Corresponding author: Guo-Liang Wang; Department of Plant Pathology, 201 Kottman Hall, 2021 Coffey Rd.; Telephone: +1.614.292.9280 (O) or +1.614.292.8231 (Lab); Fax: +1.614.292.4455; E-mail: wang.620@osu.edu

The genomic sequences of the $P i 2$-allelic locus in the rice cultivar Nipponbare and the extended region of the $P i 9$ locus in the $P i 9$-isogenic line 75-1-127 have been deposited with the EMBL/GenBank data libraries under accession numbers DQ454158 and DQ454157, respectively. The GenBank accessions of the 2,000- and 500-bp genomic fragments that bridge the three contigs to form the 66-kb 9311 supercontig (Fig. 1B) are DQ888234 and DQ888233, respectively.

* The $\boldsymbol{e}$-Xtra logo stands for "electronic extra" and indicates the HTML abstract available on-line contains supplemental material not included in the print edition. There are two additional figures and four additional tables published online. they contain a toll interleukin 1 receptor (TIR) domain that is homologous to the intracellular domain of the Drosophila toll and mammalian interleukin-1 receptors in their N-termini (Lehmann 2002; Leister 2004; Martin et al. 2003; Meyers et al. 2003). Sometimes, the non-TIR group is referred to as the coiled-coil (CC) group, because they typically have a CC domain at their $\mathrm{N}$ termini (Pan et al. 2000). It is interesting that the NBS-LRR gene repertoire is different in dicotyledonous and monocotyledonous plants (Bai et al. 2002; Meyers et al. 2003). For example, a total of 149 NBS-LRR-encoding genes were identified, of which 94 belong to the TIR-NBS-LRR class of genes and 55 to the CC-NBS-LRR gene class in Arabidopsis (Meyers et al. 2003). On the contrary, the rice genome carries approximately 500 NBS-LRR genes, none of which encode a typical TIR domain at the N-termini (Monosi et al. 2004).

In plants, it is common that the NBS-LRR genes are tightly linked at a complex locus (Hulbert et al. 2001; Leister 2004; Meyers et al. 2003). For example, all the annotated 149 NBSLRR genes in Arabidopsis are distributed as 40 singletons and 43 clusters and $73.2 \%$ of them are located in the clusters (Meyers et al. 2003). Depending on the sequence similarities between paralogs, the complex loci can be further divided into two groups, homogeneous and heterogeneous clusters (Leister 2004). In the homogeneous cluster, the paralogs are highly sequence-related. In this case, the clustering of the highly sequence-related genes is usually a result of tandem duplications of paralogous sequences (Leister 2004; Meyers et al. 2003; Richly et al. 2002). For example, the genes at the flax $P$ locus share over $95 \%$ DNA identity with one another (Dodds et al. 2001). A similar situation was also reported at the $C f-4 / 9$ locus, whereof the genes share over 92\% DNA identity (Parniske et al. 1997). On the contrary, the genes in the heterogeneous clusters are highly divergent and usually fall into different phylogenetic clades (Leister 2004). For example, the barley Mla locus carries three different families of NBS-LRR genes coding for proteins with sequence identities of less than $33 \%$ (Wei et al. 2002).

Genetic recombination via the unequal recombination events has been illustrated to be important for the generation of the $R$ gene variants and the evolution of novel resistance specificities (Ellis et al. 2000; Hulbert 1997; Smith et al. 2004). In addition to creating novel genes and reassorting them into new combinations, unequal recombination within a gene family tends to homogenize them (Hulbert et al. 2001; Leister 2004). In reality, the $R$-gene loci have maintained significant sequence polymorphism in nature, e.g., the RPP5 and RPP13 loci in Arabidopsis (Nöel et al. 1999; Rose et al. 2004). It indicates that a balance between the sequence homogenization, resulting from the frequent sequence exchange via unequal recombination, and se- 
quence diversification antagonizing the sequence homogenization has been maintained in plant $R$ gene complexes (Meyers et al. 2005). Strong diversifying selection (Mondragon-Palomino et al. 2002; Parniske et al. 1997; Rose et al. 2004), sequence divergence at the intergenic region (Parniske et al. 1997), and composition of the transposable elements (Wei et al. 2002) have been proposed to be the mechanisms mediating the sequence diversification. The ectopic and segmental duplication events, resulting in the duplicates to unlinked regions from the original one, are also proposed to be important in the maintenance of the sequence polymorphism in the $R$ gene loci (Baumgarten et al. 2003; Meyers et al. 2003).

The Pi2/9 locus contains at least four known $R$ specificities to the fungal pathogen Magnaporthe grisea. The cloned $R$ genes Pi2, Pi9, and Piz-t encode highly homologous NBSLRR proteins and are a member of a multigene family $(\mathrm{Qu}$ et al. 2006; Zhou et al. 2006). To further characterize the genomic dynamics and organization of the Pi2/9 locus, the genomic sequences of the Pi2/9 locus in five different rice cultivars were determined and compared with each other. An obvious orthologous or allelic relationship was observed at the Pi2/9 locus in which the gene members in corresponding positions in different haplotypes (orthologs) are more similar to each other than they are to the homologs within their respective haplotypes (paralogs). In addition, the $5^{\prime}$ regulatory sequence and $\mathrm{N}$-terminal intron of the paralogs within each haplotype appear to be more sequence- and size-divergent, which might be associated with the suppression of the sequence recombination among the paralogs at the Pi2/9 locus. The results on the genomic dynamics and organization of the Pi2/9 locus from this work provide new information on the evolutionary mechanism of the NBS-LRR $R$-gene complexes in plants.

\section{RESULTS}

\section{Establishment of the Pi2/9 locus} in five different rice cultivars.

We selected five rice cultivars (haplotypes) to investigate the genetic events involved in the evolution of the Pi2/9 locus. Two near-isogenic lines, C101A51 and 75-1-127, that contain the $P i 2$ and $P i 9$ resistance genes, respectively, and three susceptible rice cultivars including two indica rice cultivars, CO39 and 9311, and a japonica rice cultivar, Nipponbare, were included in this study. Given that the Pi2/9 locus in each rice haplotype contains multiple gene members, the NBS-LRR genes in the susceptible haplotypes (CO39, Nipponbare, and 9311) were designated the same way as the resistance haplotypes
(C101A51 and 75-1-127) as described previously (Qu et al. 2006; Zhou et al. 2006). As shown in Table 1, the NBS-LRR genes at each locus were named in numeric order according to their genomic positions and were discriminated by the suffixes with their respective haplotype and locus name.

The entire Pi2 locus (GenBank accession number DQ352453) was completely sequenced in the Pi2-isogenic line C101A51 from which nine NBS-LRR genes were identified (Zhou et al. 2006), and the Pi9 locus (GenBank accession number DQ285630) was partially determined in the NIP gene side in the Pi9-isogenic line 75-1-127 from which six NBS-LRR genes were identified ( $\mathrm{Qu}$ et al. 2006). In order to determine the sequence at the $P K$ gene side at the $P i 9$ locus, three bacterial artificial chromosome (BAC) clones (BAC85, BAC96, and BAC93, discussed below) were identified and completely sequenced, which were assembled into two nonoverlapping sequences. One contig sequence (GenBank accession number DQ454157), corresponding to BAC85 and BAC96, comprises three NBS-LRR genes, named $\mathrm{Nbs} 7-\mathrm{Pi}$, Nbs8-Pi9, and $\mathrm{Nbs} 9$ Pi9 (Fig. 1E). Another one does not contain any NBS-LRR gene but the $P K$ gene, representing the $3^{\prime}$ flanking sequence of the Pi9 locus (Fig. 1E). Two gaps, between Nbs6-Pi9 and Nbs7-Pi9 and between Nbs9-Pi9 and $P K$, remained at the Pi9 locus (Fig. 1E), although polymerase chain reaction (PCR) and BAC library screening approaches were tried. Therefore, the exact number of the NBS-LRR genes was not determined at the $P i 9$ locus (Fig. 1E).

The Pi2/9 locus from the susceptible rice cv. Nipponbare was also determined completely. The BAC clone OSJNBb0029O05 from the Nipponbare BAC library (Arizona Genomiocs Institute website) was completely sequenced (GenBank accession number DQ454158). The BAC sequence we obtained is identical to the sequence released by the International Rice Sequencing Consortium (International Rice Genome Sequencing Project 2005) except for a 2-nt difference. A total of seven NBS-LRR genes were identified at the Pi2/9 locus in Nipponbare (Fig. 1C). The Pi2/9 loci from another two susceptible cultivars, CO39 and 9311, were also investigated. The BAC clones 36C13 and 46L18, which correspond to the NIP and $P K$ sides of the Pi2/9 locus in CO39, respectively, were identified from the CO39 BAC library (Texas A\&M University Laboratory for Plant Genomics and GENEfinder Genomic Resource website) and were partially sequenced. From these two BACs, four NBS-LRR genes were identified at the Pi2/9 locus in CO39 (Fig. 1A). A gap between $\mathrm{Nbs} 2-\mathrm{Pi} / \mathrm{9}-\mathrm{CO} 39$ and $\mathrm{Nbs} 3-\mathrm{Pi} / \mathrm{9}$ CO39 existed after sequencing these two BACs. Using a $3.0-\mathrm{kb}$ genomic fragment of the $\mathrm{Nbs} 2-\mathrm{Pi} 2$ gene as the probe, Southern

Table 1. The Pi2/9 gene family in five rice cultivars ${ }^{\mathrm{a}}$

\begin{tabular}{|c|c|c|c|c|}
\hline $75-1-127^{b}$ & C101A51 & Nipponbare $^{c}$ & CO39 & 9311 \\
\hline Nbs1-Pi9 & Nbs1-Pi2 & Nbs1-Pi2/9-NPB & Nbs1-Pi2/9-CO39 & Nbs1-Pi2/9-9311 \\
\hline Nbs3-Pi9 & Not present & Not present & Not present & Not present \\
\hline Nbs2-Pi9/Pi9 & Nbs2-Pi2 & Nbs2-Pi2/9-NPB & Nbs2-Pi2/9-CO39 & Nbs2-Pi2/9-9311 \\
\hline Nbs4-Pi9 & Nbs3-Pi2 & Not present & $\ldots$ & $\ldots$ \\
\hline Nbs5-Pi9 & Nbs4-Pi2/Pi2 & Not present & $\ldots$ & $\ldots$ \\
\hline Nbs6-Pi9 & Not present & Not present & $\ldots$ & $\ldots$ \\
\hline$\ldots$ & Nbs5-Pi2 & Nbs3-Pi2/9-NPB & $\ldots$ & Nbs3-Pi2/9-9311 \\
\hline$\ldots$ & Nbs6-Pi2 & Nbs4-Pi2/9-NPB & $\ldots$ & Nbs4-Pi2/9-9311 \\
\hline Nbs7-Pi9 & Nbs7-Pi2 & Nbs5-Pi2/9-NPB & Nbs3-Pi2/9-CO39 & Nbs5-Pi2/9-9311 \\
\hline Nbs8-Pi9 & Nbs8-Pi2 & Nbs6-Pi2/9-NPB & Nbs4-Pi2/9-CO39 & Nbs6-Pi2/9-9311 \\
\hline Nbs9-Pi9 & Nbs9-Pi2 & Nbs7-Pi2/9-NPB & Not present & Not present \\
\hline
\end{tabular}

a The nucleotide binding site and leucine rich repeat NBS-LRR genes at the Pi2/9 locus in each haplotype were aligned in different rows based on their orthologous or allelic relationship, i.e., the orthologs were aligned in the same rows. Therefore, some genes that are not present in other cultivars were considered herein as orphan genes.

${ }^{\mathrm{b}}$ The determined Pi9 locus in the isogenic line 75-1-127 is composed of two nonoverlapping sequences, GenBank accession numbers DQ285630 and DQ454157, comprising six (Nbs1-Pi9 to Nbs6-Pi9) and three (Nbs7-Pi9 to Nbs9-Pi9) genes, respectively. Ellipses (...) indicate that the sequence gaps prevent any inference about the presence of these homologs.

${ }^{c}$ The cultivar Nipponbare was abbreviated as NPB in the gene designation. 
blot hybridization detected two hybridizing bands of the homolog when the CO39 DNA was digested with HindIII and two strong bands and one faint band when digested with BamH1 (data not shown), suggesting that at least another NBS-LRR gene highly related to $\mathrm{Nbs} 2-\mathrm{Pi} 2 / 9-\mathrm{CO} 39$ is likely present in CO39. For the Pi2/9 locus in 9311, the whole genome sequence of 9311 (Bioinformatics Technology Network website) was searched against the sequences of the Pi2/9 loci in 75-1-127, C101A51, Nipponbare, and CO39. A total of 14 contigs (AAAA02018910 to AAAA02018923) were found that were presumably located to the region based on the released data from the database. From five of them, six NBSLRR homologs were identified. Nbs1-Pi2/9-9311 and Nbs2Pi2/9-9311 were identified from contig AAAA02018910, Nbs3-Pi2/9-9311 from contig AAAA02018911, Nbs4-Pi2/99311 from contig AAAA02018915, Nbs5-Pi2/9-9311 from contig AAAA02018920, and Nbs6-Pi2/9-9311 from contig AAAA02018923. The other nine contigs were not used because some did not have obvious similarity to the Pi2/9 sequence and some were redundant with the five contigs that carried the six NBS-LRR genes. When comparing the genomic sequence of the Pi2/9 locus in CO39, the three contigs, i.e., AAAA02018915, AAAA02018920, and AAAA02018923, were further merged into one supercontig of about $66 \mathrm{~kb}$ in length and located at the $P K$ gene side (Fig. 1B). However, two small gaps were found within the supercontig, one between AAAA02018915 and AAAA02018920 and another one between AAAA02018920 and AAAA02018923. The size of these two gaps was estimated to be approximately 2,000 and 500 bp in length, respectively, based on the PCR amplification and sequencing results (data not shown). Finally, the size of the gap between AAAA02018910 and AAAA02018911 and the one between AAAA02018911 and the supercontig was not determined, due to lack of sequenced BAC clones spanning the two regions (Fig. 1B), which makes it difficult to figure out how many NBS-LRR genes are present at the Pi2/9 locus in 9311.

Comparative analysis of the Pi2/9 locus in the five rice haplotypes revealed that the NBS-LRR gene members present in the corresponding locations at the Pi2/9 locus (orthologs) are more similar to each other than they are to other genes within their respective haplotypes (paralog), which will be discussed in detail in the paragraphs below. Few orthologs are not present in all haplotypes, which are called orphan genes (Table 1). Some of them may not be true orphan genes as they may be located in the sequence gap. For example, a homolog highly related to $\mathrm{Nbs} 2$ Pi2/9-CO39 was predicted to be present in the sequence gap in

A

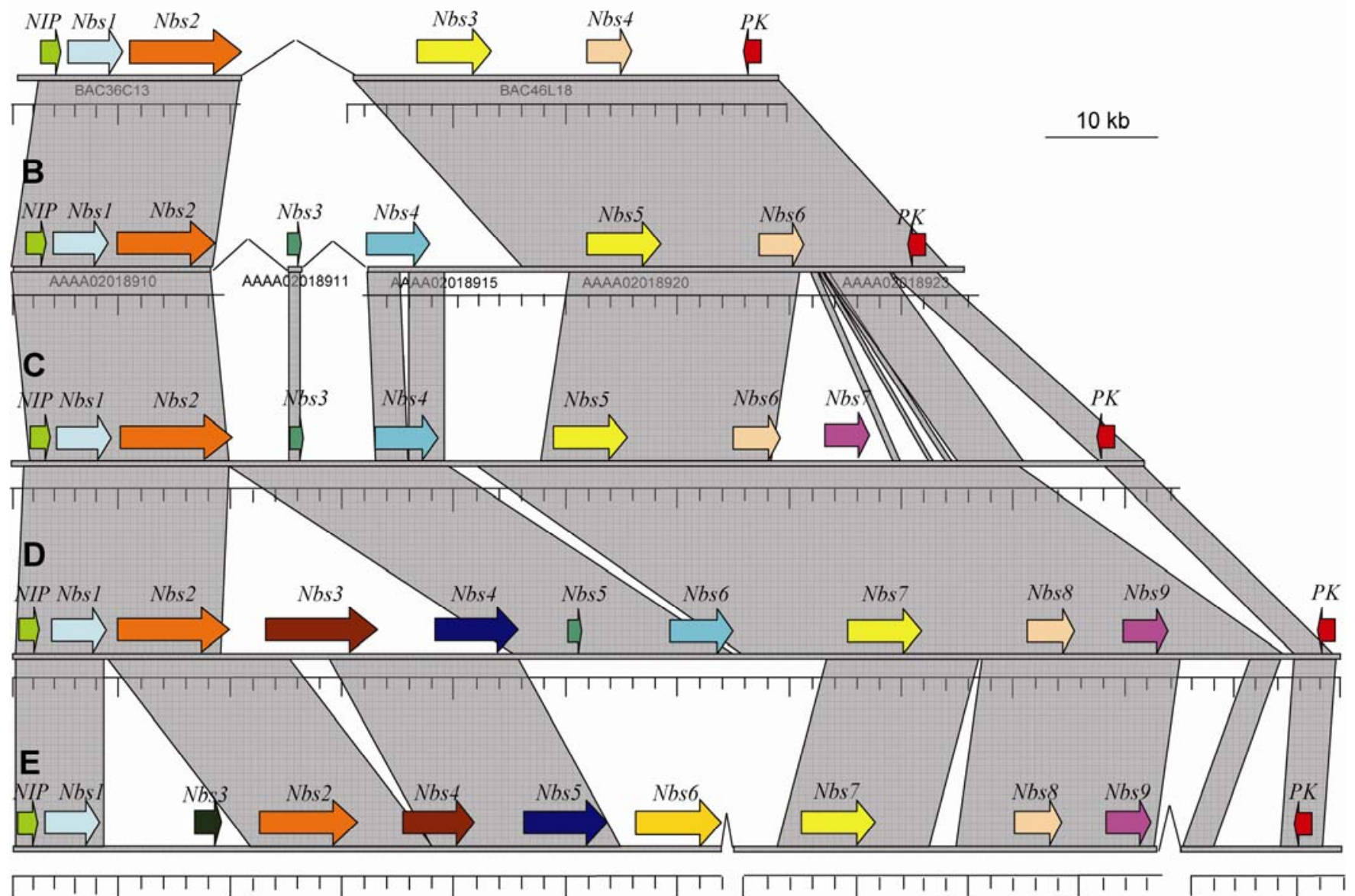

Fig. 1. Comparative analysis of the Pi2/9 locus in five rice haplotypes. The Pi2/9 gene family members, indicated by the arrows with the direction of transcription, in each haplotype were abbreviated as Nbs. The number following Nbs indicates the genomic position at the Pi2/9 locus from the NIP (nitrate-induced protein gene) to $P K$ (protein kinase gene) sides, except for $N b s 2$ and $N b s 3$ at the Pi9 locus. Different orthologs were indicated with different colors. The undetermined gaps are indicated not in scale with the line angles above. The regions showing extreme sequence identity (over $98 \%$ DNA identity overall) among different rice cultivars were shadowed. A, The Pi2/9 locus in the indica rice cultivar CO39. BAC clones 36C13 and $46 \mathrm{~L} 18$ were indicated underneath the bars representing the genomic sequence. B, The Pi2/9 locus in the indica rice cultivar 9311 . The five contigs corresponding to the region containing the nucleotide binding site and leucine-rich repeat genes were indicated underneath the bars representing the genomic sequence. C, The Pi2/9 locus in the japonica rice cultivar Nipponbare. D, The Pi2 locus in the Pi2-carrying isogenic line C101A51. E, The Pi9 locus in the Pi9-carrying isogenic line 75-1-127. 
CO39, as mentioned above. However, some orphan genes are clearly absent in other cultivars. For example, the $\mathrm{Nbs} 3-\mathrm{Pi} 9$ gene at the $P i 9$ locus was not found in the corresponding region in the other four haplotypes in which the complete sequence was determined (Table 1 and Fig. 1). Despite the different copies of NBS-LRR genes present at the Pi2/9 locus in different haplotypes, the $N I P$ and $P K$ genes were highly conserved, suggesting that they might represent the progenitor flanking sequences of the NBS-LRR gene cluster at the Pi2/9 locus (Fig. 1).

\section{The Pi2/9 locus consists}

of few transposable elements (TE).

The TE are major components in the clusters of resistance genes (Michelmore and Meyers 1998). The composition of TE, including retrotransposon, transposon, and a long terminal repeat (LTR), at the Pi2/9 locus was analyzed in three rice haplo- types, 75-1-127, C101A51, and Nipponbare. Interestingly, only a solo LTR but not typical transposons and retrotransposons was identified at the Pi2/9 locus in these three haplotypes. This suggests that TE are not abundant at the Pi2/9 locus, which is distinct from most $R$ gene complexes (Michelmore and Meyers 1998; Wei et al. 2002). Based on the conserved structure of the solo LTR, including the terminal inverted repeat and the flanking duplicated sequence, we identified a total of 11 solo LTRs in these three haplotypes. A similar feature in terms of the composition of TE was also observed at the Pi2/9 loci in the other two rice haplotypes CO39 and 9311 (data not shown).

\section{The Pi2/9 locus exhibits significant sequence variation} among the paralogs within each haplotype.

The coding sequence and genomic sequence of each NBSLRR gene at the Pi2/9 locus were used for comparative analy-

Table 2. DNA sequence identity (\%) of the gene members at the Pi2 locus

\begin{tabular}{|c|c|c|c|c|c|c|c|c|}
\hline Gene & Nbs1-Pi2 & Nbs2-Pi2 & Nbs3-Pi2 & Nbs4-Pi2 & Nbs5-Pi2 & Nbs6-Pi2 & Nbs7-Pi2 & Nbs8-Pi2 \\
\hline Nbs2-Pi2 & 71.8 & & & & & & & \\
\hline Nbs3-Pi2 & 68.2 & 61.9 & & & & & & \\
\hline Nbs4-Pi2 & 71.6 & 96.2 & 73.8 & & & & & \\
\hline Nbs5-Pi21 & 67.1 & 66.8 & 65.9 & 67.2 & & & & \\
\hline Nbs6-Pi2 & 70.5 & 95.6 & 75.3 & 96.3 & 67.2 & & & \\
\hline Nbs7-Pi2 & 70.7 & 84.2 & 72.0 & 84.1 & 66.3 & 83.5 & & \\
\hline Nbs8-Pi2 & 63.2 & 60.6 & 62.3 & 61.5 & 58.1 & 60.4 & 61.6 & \\
\hline Nbs9-Pi2 & 69.1 & 73.4 & 91.0 & 72.9 & 65.2 & 72.8 & 71.1 & 62.1 \\
\hline
\end{tabular}
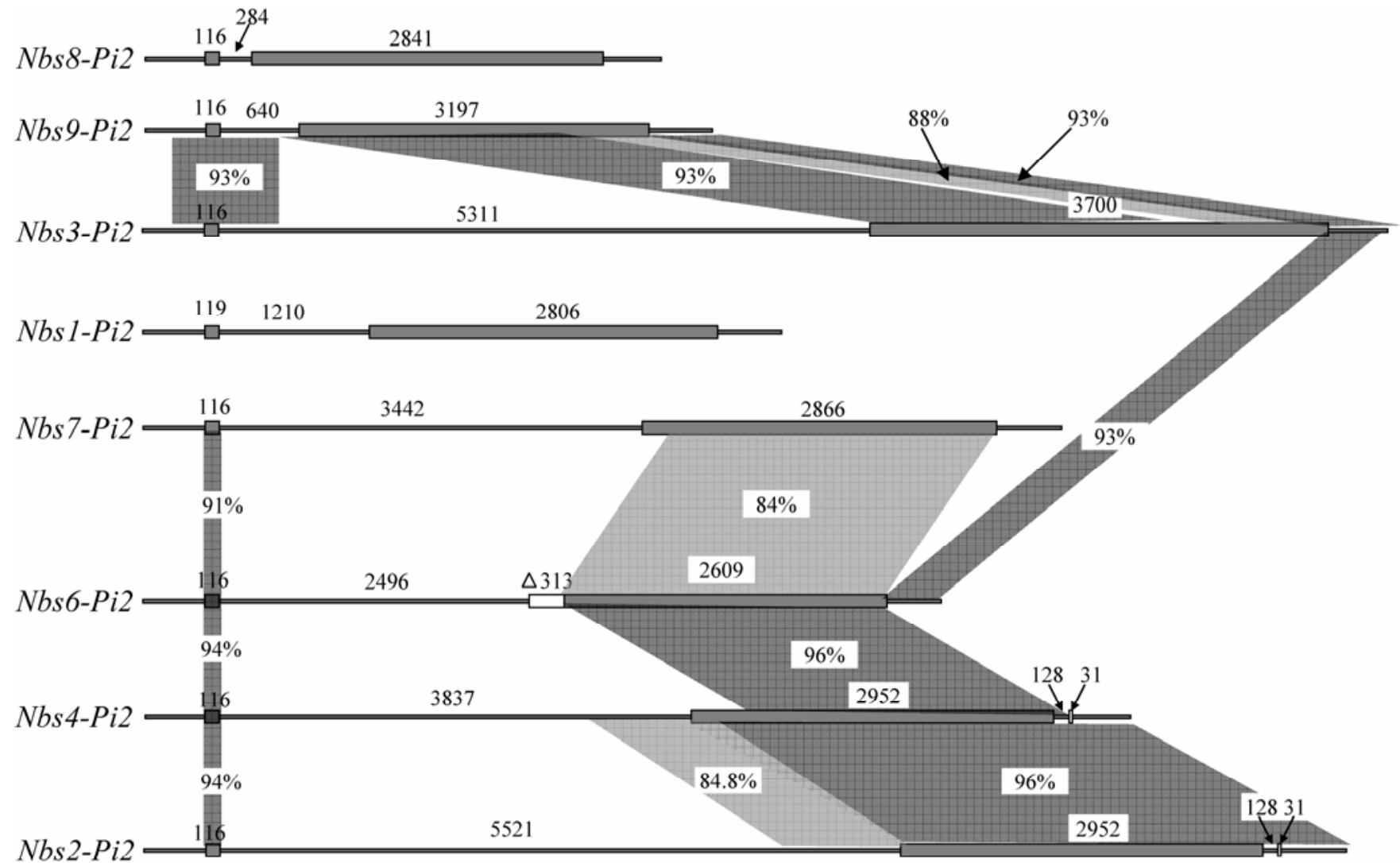

Fig. 2. Schematic representation of the contrasting level of sequence divergence between the coding and noncoding regions of the family members at the Pi2 locus. All gene members including $500 \mathrm{bp}$ of $5^{\prime}$ and $3^{\prime}$ untranslated region, except $\mathrm{Nbs5}$-Pi2, were aligned according to their sequence homology for comparison. The coding and noncoding regions were indicated in filled boxes and strings, respectively. The size of the exons and introns in base pairs were indicated by the number above. The regions sharing over $80 \%$ identity in nucleotide sequence were shadowed, with the number indicating the sequence identity level. The deletion marked in the triangle in the exon of Nbs6-Pi2 was indicated in the open box, with the number to indicate the size predicted based on the corresponding deletion of $\mathrm{Nbs} 4-\mathrm{Pi} 2$. 
sis in which a significant sequence variation was observed among paralogs within each haplotype. The paralogs share as low as $58.1 \%$ identity in nucleotides at the Pi2 locus, indicating that some gene members are significantly divergent to others (Table 2). Some paralogs, however, are highly related to each other. For example, $\mathrm{Nbs} 2-\mathrm{Pi2}, \mathrm{Nbs} 4-\mathrm{Pi2}$, and $\mathrm{Nbs6}-\mathrm{Pi2}$ at the $P i 2$ locus share over $95.6 \%$ identity in nucleotides to each other (Table 2). A similar feature in terms of their sequence divergence was also observed among the paralogs at the Pi2/9 locus in the other four rice haplotypes (data not shown).

Furthermore, the noncoding sequence appears to be more divergent than the coding sequence at the Pi2/9 locus. Most of the paralogs do not share apparent sequence identity in their $5^{\prime}$ regulatory sequences, including the $5^{\prime}$ untranslated region sequence and promoter region, even though they share extreme sequence identity in their coding sequences. For example, $\mathrm{Nbs} 2-\mathrm{Pi2}, \mathrm{Nbs} 4-\mathrm{Pi2}$, and Nbs6-Pi2 at the Pi2 locus share as high as $96 \%$ nucleotide sequence identity in their coding region to each other, but they do not share any sequence similarity in their $5^{\prime}$ regulatory sequence (Fig. 2). In addition to the high divergence at the $5^{\prime}$ regulatory sequences, the N-terminal intron of the paralogs at the Pi2/9 locus in each haplotype always have different sizes and low or even no sequence similarity to each other (Fig. 2). The size of the N-terminal intron ranges from $284 \mathrm{bp}$ for $\mathrm{Nbs} 8-\mathrm{Pi} 2$ to $5,521 \mathrm{bp}$ for $\mathrm{Nbs} 2-\mathrm{Pi} 2$ at the Pi2 locus, $267 \mathrm{bp}$ for $N b s 6-P i 2 / 9-N P B$ to 5,364 bp for Nbs2-Pi2/9-NPB at the Pi2/9 locus in Nipponbare, 153 bp for $\mathrm{Nbs3}-\mathrm{Pi} 9$ to 5,362 bp for $\mathrm{Nbs2}-\mathrm{Pi} 9$ at the $\mathrm{Pi} 9$ locus. It is evident that the insertion of some TE may have contributed to the size divergence of N-terminal introns such as the solo LTRs in Nbs3-Pi2, Nbs4-Pi9, and Nbs6-Pi9. Moreover, the level of the sequence identity of the $\mathrm{N}$-terminal introns is much lower than that in the coding sequence in most of the paralogs at the Pi2/9 locus. For example, Nbs2-Pi2 and Nbs6-Pi2 share 96\% nucleotide sequence identity in the coding region but no sequence similarity to each other in the N-terminal intron (Fig. 2).

In addition to the significant sequence and size divergence of the noncoding regions in the paralogs at the Pi2/9 locus, the intergenic sequence is also highly divergent at the Pi2/9 locus within each haplotype. No apparent sequence similarity of the intergenic regions between two neighboring paralogs was identified at the locus.

\section{All the NBS-LRR genes at the Pi2/9 locus from five rice haplotypes have the same phase- 2 introns at the $\mathrm{N}$-terminus.}

It was demonstrated that there is a good agreement between the evolutionary relationship and the intron position and phase in the NBS-LRR gene families in plants (Bai et al. 2002; Meyers et al. 2003). Both Pi2 and Pi9 (corresponding to Nbs4$\mathrm{Pi} 2$ and $\mathrm{Nbs} 2-\mathrm{Pi}$, respectively, in this study) have a phase-2 intron, which interrupts a codon between the second and third bases (Sharp 1981) at the N-terminus (Qu et al. 2006; Zhou et al. 2006). This intron position is quite unique from most of the NBS-LRR gene families in rice (Bai et al. 2002; Qu et al. 2006; Zhou et al. 2006). To further investigate whether it is conserved in other NBS-LRR genes at the Pi2/9 locus, intron positions and phases were investigated in all the genes at the $\mathrm{Pi2} / 9$ locus from five haplotypes, except $\mathrm{Nbs} 5-\mathrm{Pi} 2$ and $\mathrm{Nbs} 3$ $P i 2 / 9-N P B$, for they are severely truncated. We found that all the NBS-LRR genes at the Pi2/9 locus have introns at the $\mathrm{N}$ terminus (Fig. 2, only the genes at the Pi2 locus were illustrated). Moreover, their first exons are either 116 or $119 \mathrm{bp}$ in length, which ensures the following $\mathrm{N}$-terminal intron to retain the phase-2 intron (Fig. 2, only the genes at the Pi2 locus were illustrated). The conserved intron position and phase of the NBS-LRR genes at the Pi2/9 locus strongly suggest that they might be evolved from a common progenitor carrying the same gene architecture.

\section{The NBS-LRR genes at the Pi2/9 locus belong to the same phylogenetic clade.}

We have demonstrated that all the NBS-LRR genes at the Pi2/9 locus conserve the same intron position and phase even though they show a significant sequence divergence within the same haplotype. In order to further determine the evolutionary relationship of the NBS-LRR genes at the Pi2/9 locus with their homologs located at other loci in the genome, a phylogenetic analysis of the NBS-LRR genes at the Pi2/9 and other loci was conducted. We used the sequences of the NBS-LRR genes at the Pi2/9 locus to search against the nonredundant protein NCBI database and found many homologs located outside the Pi2/9 locus. Interestingly, most of them share lower than $58 \%$ identity in nucleotide sequence to the NBS-LRR genes at the Pi2/9 locus, except for P0425F02.21 and P0036F10.15 (GenBank accession numbers BAA90798 and BAD68775, respectively; data not shown). This finding suggests that the NBS-LRR genes at the Pi2/9 locus are more sequencerelated to each other than they are to their homologs at other loci. The neighbor-joining tree based on the NBS sequences of the NBS-LRR genes at the Pi2/9 locus as well as their homologs at other loci was then constructed. It clearly showed that the NBS-LRR genes at the Pi2/9 locus were grouped together in the same large clade from the Pib gene as well as other homologs (Fig. 3). However, both P0425F02.21 and P0036F10.15 were clustered within the same large clade. Interestingly, both P0425F02.21 and P0036F10.15 have the same intron position and phase as the NBS-LRR genes at the Pi2/9 locus. On the contrary, the $P i b$ gene has the intron in the NBS region, representing the common intron position of the majority of the NBS-LRR genes in rice (Bai et al. 2002; Wang et al. 1999). Taken together, we conclude that the NBS-LRR genes at the Pi2/9 locus belong to the same gene subfamily, which is consistent with the above finding based on their conserved intron position and phase.

\section{The size of the intron at the $\mathrm{N}$-terminus correlates to the evolutionary relationship} of the genes at the Pi2/9 locus within each haplotype.

In the neighbor-joining tree based on the NBS sequences, we found that more divergent gene members usually have a smaller size of the N-terminal intron (Fig. 3). For example, Nbs3-Pi9, which has the smallest intron at the N-terminus at only 153 bp in length, is the most divergent one at the Pi2/9 locus (Fig. 3). Furthermore, the genes in the inner clades always have shorter branch lengths compared with the ones in outer clades, indicating that they might have evolved more recently. On the contrary, the genes in the outer clades always have longer branch lengths compared with the ones in inner clades, indicating that they might have evolved a long time ago. The highly positive correlation between the N-terminal intron size and the evolutionary relationship of the NBS-LRR genes at the Pi2/9 locus was also observed in the neighborjoining tree rebuilt in the individual haplotypes. A minor exception was observed, however, at the Pi2 locus, which may be attributed to the high sequence identity among $\mathrm{Nbs} 2-\mathrm{Pi}$, $\mathrm{Nbs} 4-\mathrm{Pi2}$, and Nbs6-Pi2. The deletion in Nbs6-Pi2 (Fig. 2), which might result in its intron size reduction, would be another reason for the relationship between $\mathrm{Nbs6-Pi2}$ and $\mathrm{Nbs} 7$ $P i 2$. A similar situation was also observed at the Pi2/9 locus in Nipponbare. The good correlation between the size of the intron at the $\mathrm{N}$-terminus and the evolutionary relationship of the NBS-LRR genes at the Pi2/9 locus may reflect the order of their occurrence during the course of the evolution. 


\section{Diversifying selection was detected predominantly in the LRR domains.}

Positive selection has been detected in most $R$ gene sequences, which is beneficial for the fixation of the sequence diversification and, therefore, for the generation of novel resistance specificities to the changing pathogens. Three pairs of maximum likelihood models of codon substitution, M3/M0, M8/M7, and M2/M1 were employed to investigate the sites subject to positive selection (Yang 1997; Yang et al. 2000) at the Pi2/9 locus. The discrete model M3 with three-site class suggested that about $10 \%$ of the amino-acid sites were under diversifying selection with $\omega_{1}=4.87$, whereas about $0.5 \%$ of amino acid sites were under strong diversifying selection with $\omega_{2}=30.1$. The likelihood ratio test for comparing M3 with M0 is $2 \Delta \mathrm{L}=334.14$, which is much greater than the $\chi^{2}$ critical value (13.28 at the $1 \%$ significance level, with degrees of freedom =4). This indicates that the discrete model M3 fits the data significantly better than the neutral model M0, which does not allow for the presence of diversifying selection sites with $\omega>1$. A total of 36 amino-acid sites were identified, which are implicated as being under diversifying selection, with greater than $99 \%$ confidence under the discrete model M3. When the positions of these sites in the encoded proteins were plotted, a disproportionate number of sites were located in the LRR region (Fig. 4). Of all these 36 sites, four are located in the N-terminus, two are located in the NBS domain, and five are located in the C-terminus. The other 25 sites were confined in the LRR region, constituting $70 \%$ of the total sites. Furthermore, of the 25 sites in the LRR region, 14 were confined in the xxLxLxx motif.

\section{The orthologs at the Pi2/9 locus}

in the five different haplotypes are highly conserved in both coding and noncoding sequences.

In contrast to the significant sequence variation of the paralogs at the Pi2/9 locus within each haplotype, the orthologs in five haplotypes share extreme sequence identity to each other. For example, $\mathrm{Nbs} 1-\mathrm{Pi} 2$ shares approximately $99 \%$ sequence identity in nucleotides to its orthologs in the other four rice haplotypes but shares only 63.2 to $71.8 \%$ sequence identity to the paralogs at the same locus (Fig. 1 and Table 2). Distinct from the asymmetric sequence similarity level between the coding and noncoding regions present in the paralogs, the orthologs share similar levels of sequence identity in noncoding and coding sequences. For example, the $5^{\prime}$ regulatory sequence and intron of the Nbs 1-Pi2 share approximately $99 \%$ sequence identity in nucleotides, the same level as that in the coding sequence, to its orthologs in the other four rice haplotypes (Fig. 1). Moreover,

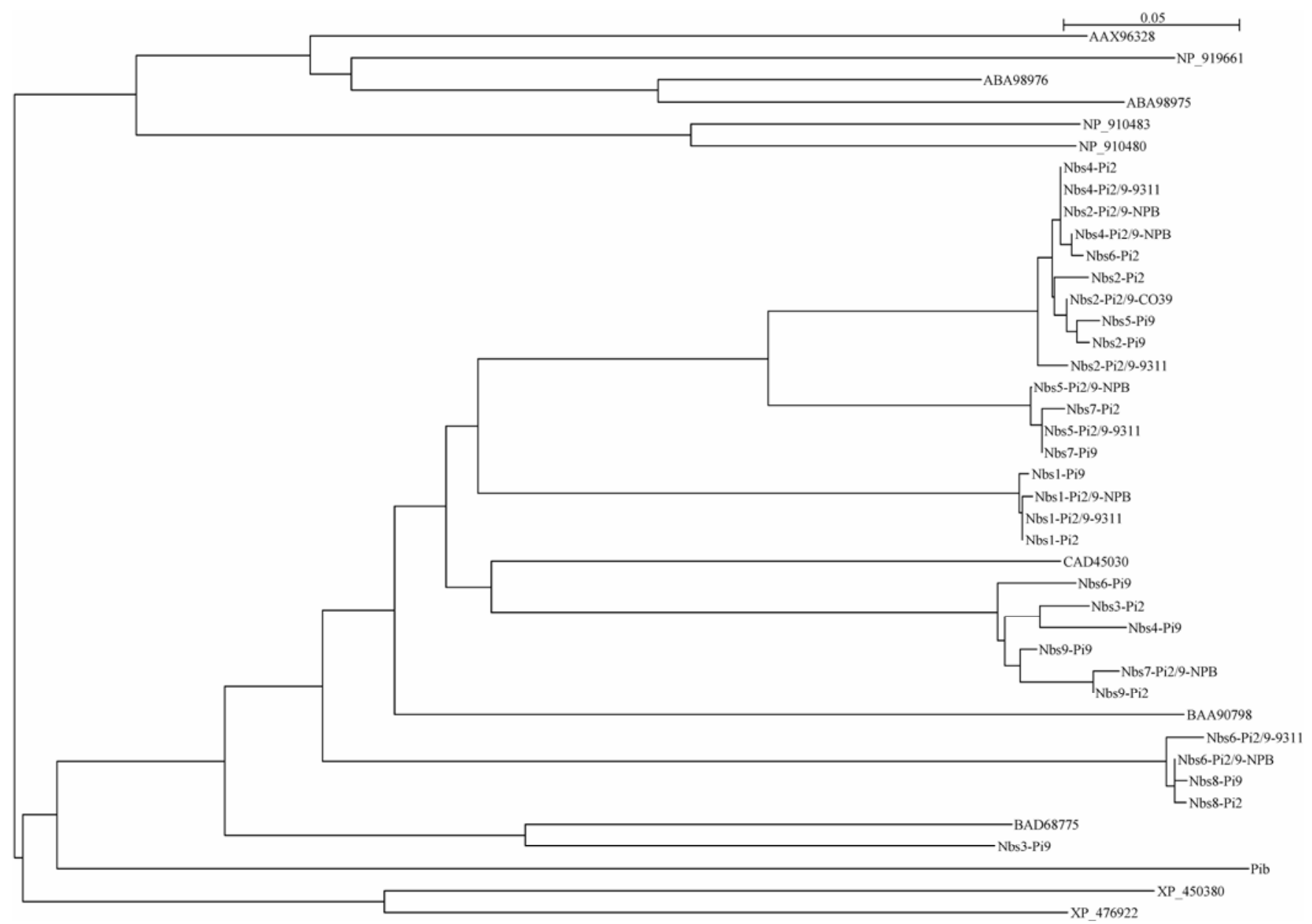

Fig. 3. Cladistic analysis of the Pi2/9 gene family members. All the Pi2/9 gene family members, except for Nbs5-Pi2, Nbs3-Pi2/9-NPB, Nbs3-Pi2/9-9311, Nbs1-Pi2/9-CO39, Nbs3-Pi2/9-CO39, and Nbs4-Pi2/9-CO39, in five rice cultivars were included in the cladistic analysis. The reason that the mentioned members were not included in the analysis is due to the difficulties in retrieving their putative nucleotide binding site (NBS) sequences caused by sequence deletions or sequence gaps. For the rice homologs of the Pi2/9 gene family located outside the Pi2/9 locus, we selected those that share over $36 \%$ identity in amino-acid sequence in the BLAST search against the Pi2/9 NBS domain. The GenBank accession numbers were used to represent these homologs except for the Pib gene (GenBank accession number BAA76281). The sequences of the NBS domain of the analyzed genes were used for generating a neighborjoining tree. Multiple sequence alignment was done using the Clustalx program (Thompson et al. 1997), and the derived neighbor-joining tree was viewed using the NJplot program (Perriere and Gouy 1996). 
the intron size is almost the same between $\mathrm{Nbs} 1-\mathrm{Pi} 2$ and its orthologs. The intergenic sequence present at the corresponding locations in different haplotypes at the Pi2/9 locus is also highly similar among haplotypes, indicating that a good sequence synteny is maintained at the Pi2/9 locus in the five cultivars.

\section{The $P i 2 / 9$ gene family is highly conserved} in gramineae species.

To examine the extent of the sequence similarity of the NBSLRR genes at the Pi2/9 locus to the orthologs in other cereals, the deduced protein sequences of the NBS-LRR genes, which represent most of the ortholog groups at the Pi2/9 locus, were used to search against both expressed sequence tag (EST) and genomic sequences of cereals available in the public databases. A total of 13 EST or mRNA sequences, sharing significant sequence identity to the genes at the Pi2/9 locus, were retrieved from sorghum, barley, wheat, and sugarcane. Nine different resistance gene analogs (RGA) were then obtained by sequence assembly, most of which are not full length. Pairwise sequence comparison revealed that they share a significant sequence identity in nucleotide sequence to the genes at the Pi2/9 locus, ranging from $57.4 \%$ for $\mathrm{Nbs} 8-\mathrm{Pi} 2 / \mathrm{SO} 1$ to $81.6 \%$ for $\mathrm{Nbs} 1-\mathrm{Pi} 2 / \mathrm{SB} 2$. To confirm that the RGA we obtained are the true orthologs of the Pi2/9 gene family in the respective species, a reverse search, using their deduced protein sequences against the rice genomic sequence, was conducted. The results indicated that the Pi2/9 gene family is their most related sequences. Both $H V I$ and $S B 1$ were further included for the phylogenetic analysis with the $P i 2 / 9$ gene family as well as other homologs in rice. It was found that both $H V 1$ and $S B 1$ were clustered within the Pi2/9 gene family. The closer evolutionary relationship of the Pi2/9 gene family with the RGA from different gramineae species than to the NBS-LRR genes in rice indicates that the Pi2/9 gene family might have evolved prior to speciation of different gramineae species.

\section{DISCUSSION}

The progenitor of the NBS-LRR genes at the Pi2/9 locus may have a small $\mathrm{N}$-terminal intron.

Tandem, ectopic, and segmental duplication have been proposed for the genetic events in the evolution of the NBS-LRR gene complex in Arabidopsis and rice (Baumgarten et al. 2003;
Leister 2004; Meyers et al. 2003). Among them, tandem duplication via unequal recombination was demonstrated to be the major genetic event (Meyers et al. 2003; Leister 2004). Since the intron phase is usually conserved due to the requirement for the concordant change of both sides of each intron in order to maintain the reading frame (Long and Deutsch 1999), the conserved phase-2 intron at the N-terminus present in all the NBS-LRR genes at the Pi2/9 locus strongly indicated that their progenitor should also have the intron with the same phase and position. Good agreement between the intron position and their evolutionary relationship was indeed observed in the NBS-LRR genes in Arabidopsis and rice (Bai et al. 2002; Meyers et al. 2003). Furthermore, all the NBS-LRR genes at the Pi2/9 locus were clustered into the same phylogenetic clade. Therefore, we speculated that all the NBS-LRR genes at the Pi2/9 locus most likely had evolved by tandem duplication from a single ancestral NBSLRR gene containing a phase- 2 intron at the N-terminus. However, we found that the sequence of the $\mathrm{N}$-terminal intron as well as the $5^{\prime}$ regulatory region of the paralogs at the Pi2/9 locus are very different from each other, making it difficult to speculate the chronology of their occurrence. On the contrary, it was clearly demonstrated that the genes with larger N-terminal intron occurred more recently than the ones with smaller N-terminal introns at the Pi2/9 locus. We thus speculate that the progenitor of all the NBS-LRR genes at the contemporary Pi2/9 locus should contain a small intron.

\section{Sequence divergence and N-terminal intron expansion} play a major role in shaping the $\mathrm{Pi} / 9$ locus.

We have demonstrated that tandem duplication via unequal recombination is the major evolutionary force driving the generation of the multiple NBS-LRR genes at the Pi2/9 locus. Sequence divergence of the duplicates is always the subsequent genetic event after gene duplication, which is usually associated with their functional divergence (Zhang 2003; Moore and Purugganan 2005). We found that the NBS-LRR genes share as low as $58.1 \%$ identity in nucleotide sequence at the Pi2 locus. A similar feature of sequence divergence was also observed at the Pi2/9 locus in other rice haplotypes. Furthermore, the 5' regulatory sequences of the paralogs are highly divergent to each other at the Pi2/9 locus. Taken together, we concluded that sequence divergence after the gene duplication facilitated the evolution of the Pi2/9 locus.

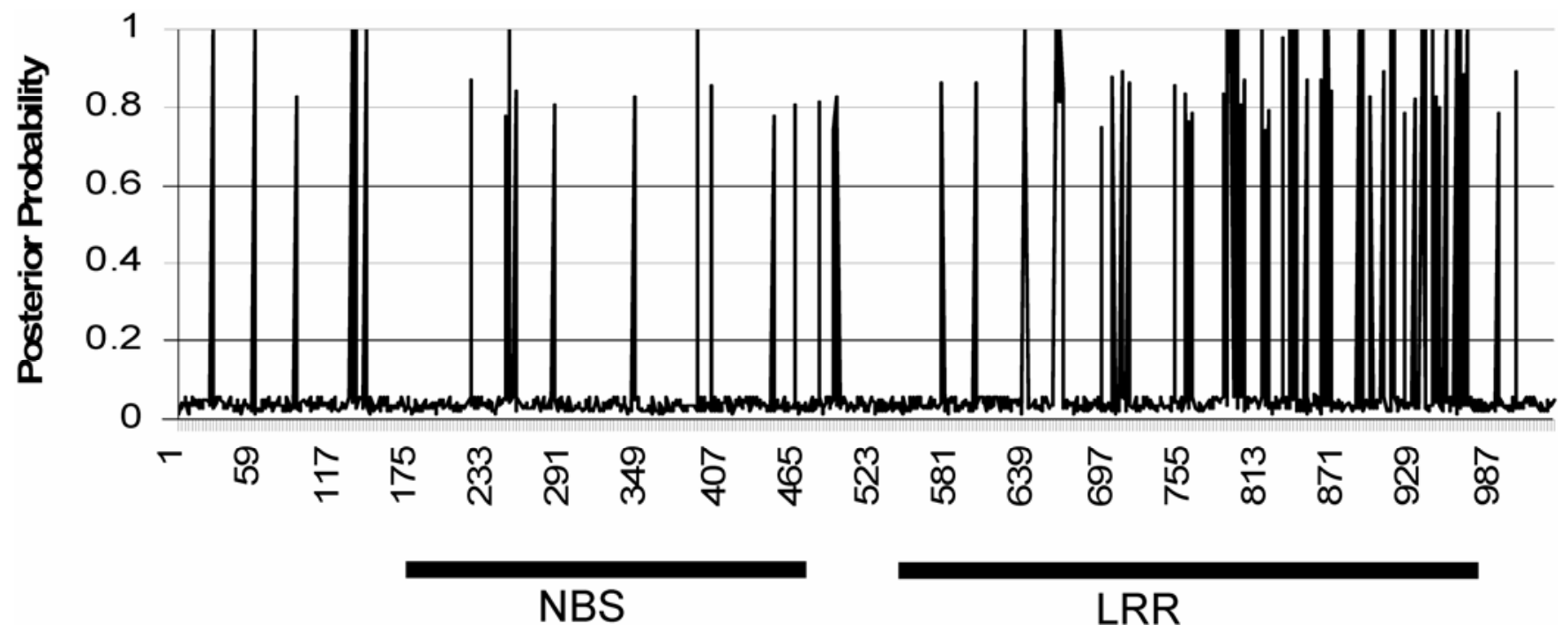

Fig. 4. Diversifying selection was predominantly distributed at the leucine-rich repeat (LRR) regions of the Pi2/9 gene family members. Posterior probabilities for sites under the selection model M3 along the Pi2/9 gene family members are shown in bars. The nucleotide binding site and LRR domains were indicated underneath the $x$ axis. 
In addition to these two mechanisms, we proposed another possible mechanism by which the Pi2/9 locus evolved. It seems that the $\mathrm{N}$-terminal intron has played a critical role in the evolution of the Pi2/9 locus. The size of the N-terminal intron is quite divergent, representing unique genomic dynamics from most of the NBS-LRR gene complex. As we discussed above, the progenitor of the NBS-LRR genes most likely consisted of a small $\mathrm{N}$-terminal intron. Moreover, we found a good correlation between the N-terminal intron size and the evolutionary relationship of the paralogs at the Pi2/9 locus. Therefore, we believe that the intron expansion may have played a major role in shaping the Pi2/9 locus, as suggested in other eukaryotic organisms (Nielsen et al. 2004; Roy and Gilbert 2005). In addition, we believe that the intron expansion might also be associated with the evolution of the novel resistance because the three cloned $R$ genes, $P i 2, P i 9$, and Piz-t, all contain a large $\mathrm{N}$-terminal intron.

\section{The extreme divergence in the $5^{\prime}$ regulatory region and $\mathrm{N}$-terminal intron facilitates the maintenance of an obvious orthologous relationship at the Pi2/9 locus.}

It is evident that the inter- and intragenic exchanges via unequal recombination play a major role in the generation of new resistance specificities in many NBS-LRR gene complexes (Nöel et al. 1999; Rose et al. 2004; Smith et al. 2004; Wei et al. 2002). However, unequal recombination will usually lead to sequence homogenization, which, in turn, will hinder the generation of novel resistance specificity (Leister 2004). In reality, the $R$ gene locus usually exhibits a significant sequence polymorphism in different haplotypes, suggesting a balance between sequence homogenization and sequence diversification has been maintained during the evolution of the $R$ gene loci (Meyers et al. 2005). The strong positive selection is one of the mechanisms to prevent the process of sequence homogenization (Bergelson et al. 2001; Leister 2004). Sequence divergence at the intergenic region and clustering of the TE are two more mechanisms by which intergenic sequence recombination was suppressed (Parniske et al. 1997; Wei et al. 2002). Baumgarten and associates (2003) presented another mechanism, by which the duplication of NBS-LRR sequences to unlinked chromosomal regions placed them out of reach of intergenic sequence exchanges.

We have demonstrated that a strong positive selection has predominantly acted on the LRR regions of the Pi2/9 gene family members and that a significant sequence divergence is present among the paralogs and intergenic sequences at the Pi2/9 locus within each haplotype. In addition to these two mechanisms, we found that the $5^{\prime}$ regulatory sequence and $\mathrm{N}$ terminal introns are extremely diversified in sequence and size among the paralogs at the Pi2/9 locus within a single haplotype. It has been demonstrated that intron size negatively correlates with the frequency of the recombination in Drosophila melanogaster and vertebrates (Carvalho and Clark 1999; Comeron and Kreitman 2000; Duret et al. 1995). In this context, the rate of recombination decreases in the region containing large introns. At the Pi2/9 locus, the genes that are highly similar to other paralogs always have the larger $\mathrm{N}$-terminal introns, whereas the genes that are more divergent always have the smaller N-terminal introns. This finding suggested that the large intron may act as a "recombination suppressor" to reduce the rate of the sequence exchange between the closely related paralogs. Furthermore, the 5' region of the genes has been demonstrated to be the initial point for the recombination, suggesting that the $5^{\prime}$ region is critical for the meiotic recombination (Wu and Lichten 1994; Xu et al. 1995). Therefore, greater sequence divergence in the $5^{\prime}$ region may lead to a reduced likelihood of meiotic recombinabtion. We observed a significant sequence divergence at the $5^{\prime}$ regulatory region as well as the
N-terminal intron at the Pi2/9 locus, which may interfere with meiotic mispairing and thus suppress the recombination between the paralogs at the locus. Taken together, our results show that the extreme divergence of the 5' regulatory and the $\mathrm{N}$-terminal intron sequences may play an important role in the suppression of the recombination between the paralogs at the Pi2/9 locus, which, in turn, facilitates the maintenance of the orthologous relationship at the locus.

\section{MATERIALS AND METHODS}

\section{Identification of the BAC clones covering the Pi2/9 locus from 75-1-127, Nipponbare, and CO39.}

To identify the BAC clones spanning the Pi2/9 locus in Nipponbare, the end sequences of Nipponbare BAC libraries (Arizona Genomics Institute website) were BLAST-searched using the Pi2 genomic sequence (GenBank accession number DQ352453) as the query. The BAC clone OSJNBb0029O05 was found to cover the Pi2/9 locus, as its two end sequences match the $P i 2$ genomic sequence. To extend the sequence to the $3^{\prime}$ end of the Pi9 locus, the BAC library derived from the Pi9-carrying rice cultivar 75-1-127 was used for the screening (Liu et al. 2002). The Sp6 end of the BAC clone BAC6 (Zhou et al. 2006) and the T7 end of the Nipponbare BAC clone OSJNBb0029O05 were used to screen the BAC library, using the PCR screening method as described previously (Zhou et al. 2006). Two BAC clones, BAC96 and BAC93, were identified and used for further chromosome-walking. Another BAC clone, BAC85, was also identified, which is overlapped with BAC96 but not with BAC93. For the BAC clones from CO39, the NIP sequence at the Pi2 locus and T7 end of the BAC clone OSJNBb0029O05 were used to screen the CO39 BAC library (Texas A\&M University Laboratory for Plant Genomics and GENEfinder Genomic Resource website), using the same method described previously (Zhou et al. 2006). Two BAC clones, BAC36C13 and BAC46L18, were identified that did not overlap each other.

DNA sequencing, sequence assembly, and gap filling.

The BAC clones OSJNBb0029005, BAC96, BAC85, and BAC93 were completely sequenced, while both BAC36C13 and BAC46L18 were partially sequenced. The shotgun library construction and subclone sequencing were conducted at the DuPont Crop Genetics Genomic Facility in Delaware. The sequence reads were assembled with the Phred and Phrap software packages (Ewing and Green 1998; Ewing et al. 1998) and were edited with the Consed program (Gordon et al. 1998). At eight to ten time redundancies, internal low-quality regions of each contig were treated as gaps, which were further filled using the primer-walking procedure as described previously (Zhou et al. 2001).

\section{Computation analysis of the sequences.}

The genomic sequence was annotated by using the gene prediction program Fgenesh (Softberry website) and was manually edited by a homology search against available databases. The genomic sequence at the Pi2 locus was used as reference for the annotation. The Matcher program (Institut Pasteur website) was used for pairwise sequence analysis. The nucleotide sequence, instead of the proteins sequence, was used for comparison because some gene members do not contain the entire coding sequence. The sequence of the NBS domain of each gene was retrieved with the BLAST2 program, using the NBS sequence of the Pi2 protein. All the NBS sequences were used for phylogenetic analysis by the Clustalx program (Thompson et al. 1997). The phylogenetic tree was viewed either with Njplot (Perriere and Gouy 1996) or Mega 2 (Kumar et al. 2001) programs. The entire coding sequences of six Pi2/9 gene family members 
(Nbs2-Pi2, Nbs4-Pi2, Nbs2-Pi2/9-NPB, Nbs2-Pi2/9-CO39, $\mathrm{Nbs} 2-\mathrm{Pi}$, and $\mathrm{Nbs} 5-\mathrm{Pi}$ ) were used for the phylogenetic analysis by maximum likelihood (PAML) analysis to detect the sites subject to the diversifying selection (Yang 1997; Yang et al. 2000).

\section{ACKNOWLEDGMENTS}

This project was in part supported by a grant from DuPont Inc. and an Industry Matching grant of the SEEDS program of the Ohio Agriculture Research and Development Center (OARDC).

\section{LITERATURE CITED}

Bai, J., Pennill, L. A., Ning, J., Lee, S. W., Ramalingam, J., Webb, C. A., Zhao, B., Sun, Q., Nelson, J. C., Leach, J. E., and Hulbert, S. H. 2002. Diversity in nucleotide binding site-leucine-rich repeat genes in cereals. Genome Res. 12:1871-1884.

Baumgarten, A., Cannon, S., Spangler, R., and May, G. 2003. Genomelevel evolution of resistance genes in Arabidopsis thaliana. Genetics 165:309-319.

Bergelson, J., Kreitman, M., Stahl, E. A., and Tian, D. 2001. Evolutionary dynamics of plant $R$-genes. Science 292:2281-2285.

Carvalho, A. B., and Clark, A. G. 1999. Intron size and natural selection. Nature 401:344.

Comeron, J. M., and Kreitman, M. 2000. Population, evolutionary and genomic consequences of interference selection. Genetics 156:1175-1190.

Duret, L., Mouchiroud, D., and Gautier, C. 1995. Statistical analysis of vertebrate sequences reveals that long genes are scarce in GC-rich isochores. J. Mol. Evol. 40:308-317.

Dodds, P. N., Lawrence, G. J., and Ellis, J. G. 2001. Six amino acid changes confined to the leucine-rich repeat beta-strand/beta-turn motif determine the difference between the $P$ and $P 2$ rust resistance specificities in flax. Plant Cell 13:163-178.

Ellis, J., Dodds, P., and Pryor, T. 2000. Structure, function and evolution of plant disease resistance genes. Curr. Opin. Plant Biol. 3:278-284.

Ewing, B., and Green, P. 1998a. Base calling of automated sequencer traces using Phred. II. Error probabilities. Genome Res. 8:186-194.

Ewing, B., Hillier, L., Wendl, M., and Green, P. 1998b. Base calling of automated sequencer traces using Phred. I. Accuracy assessment. Genome Res. 8:175-185.

Gordon, D., Abajian, C., and Green, P. 1998. Consed, a graphical tool for sequence finishing. Genome Res. 8:195-202.

Hulbert, S. H. 1997. Structure and evolution of the rpl complex conferring rust resistance in maize. Annu. Rev. Phytopathol. 35:293-310.

Hulbert, S. H., Webb, C. A., Smith, S. M., and Sun, Q. 2001. Resistance gene complexes: Evolution and utilization. Annu. Rev. Phytopathol. 39:285-312.

International Rice Genome Sequencing Project 2005. The map-based sequence of the rice genome. Nature 436:793-800.

Kumar, S., Tamura, K., Jakobsen, I. B., and Nei, M. 2001. MEGA2: Molecular evolutionary genetics analysis software. Bioinformatics 17:1244-1245.

Lehmann, P. 2002. Structure and evolution of plant disease resistance genes. J. Appl. Genet. 43:403-414.

Leister, D. 2004. Tandem and segmental gene duplication and recombination in the evolution of plant disease resistance gene. Trends Genet. 20:116-122.

Liu, G., Lu, G., Zeng, L., and Wang, G. L. 2002. Two broad-spectrum blast resistance genes, $P i 9(t)$ and $P i 2(t)$, are physically linked on rice chromosome 6. Mol. Genet. Genomics 267:472-480.

Long, M., and Deutsch, M. 1999. Association of intron phases with conservation at splice site sequences and evolution of spliceosomal introns. Mol. Biol. Evol. 16:1528-1534.

Martin, G. B., Bogdanove, A. J., and Sessa, G. 2003. Understanding the function of plant disease resistance proteins. Annu. Rev. Plant Biol. 54:23-61.

Meyers, B. C., Kozik, A., Griego, A., Kuang, H., Michelmore, R. W. 2003. Genome-wide analysis of NBS-LRR-encoding genes in Arabidopsis. Plant Cell 15:809-834.

Meyers, B. C., Kaushik, S., and Nandety, R. S. 2005. Evolving disease resistance genes. Curr. Opin. Plant Biol. 8:129-134.

Michelmore, R. W., and Meyers, B. C. 1998. Clusters of resistance genes in plants evolve by divergent selection and a birth-and-death process. Genome Res. 8:1113-1130.

Mondragon-Palomino, M., Meyers, B. C., Michelmore, R. W., and Gaut, B. S. 2002. Patterns of positive selection in the complete NBS-LRR gene family of Arabidopsis thaliana. Genome Res. 12:1305-1315.
Monosi, B., Wisser, R. J., Pennill, L., and Hulbert, S. H. 2004. Full-genome analysis of resistance gene homologues in rice. Theor. Appl. Genet.109:1434-1447.

Moore, R. C., and Purugganan, M. 2005. The evolutionary dynamics of plant duplicate genes. Curr. Opin. Plant Biol. 8:122-128

Nielsen, C. B., Friedman, B., Birren, B., Burge, C. B., and Galagan, J. E. 2004. Patterns of intron gain and loss in fungi. PloS Biol. 2:2234-2242.

Noël, L., Moores, T. L., van Der Biezen, E. A., Parniske, M., Daniels, M. J., Parker, J. E., and Jones, J. D. G. 1999. Pronounced intraspecific haplotype divergence at the RPP5 complex disease resistance locus of Arabidopsis. Plant Cell 11:2099-2112.

Pan, Q., Wendel, J., and Fluhr, R. 2000. Divergent evolution of plant NBSLRR resistance gene homologues in dicot and cereal genome. J. Mol Evol. 50:203-213.

Parniske, M., Hammond-Kosack, K. E., Golstein, C., Thomas, C. M., Jones, D. A., Harrison, K., Wulff, B. B. H., and Jones, J. D. G. 1997. Novel disease resistance specificity result from sequence exchange between tandemly repeated genes at the $C f-4 / 9$ locus of tomato. Cell 91:821-832.

Perrière, G., and Gouy, M. 1996. WWW-Query: An on-line retrieval system for biological sequence banks. Biochimie 78:364-369.

Qu, S., Liu, G., Zhou, B., Bellizzi, M., Zeng, L., Han, B., Wang, G. L. 2006. The broad-spectrum blast resistance gene $P i 9$ encodes an NBSLRR protein and is a member of the multiple family in rice. Genetics 172:1901-1914.

Richly, E., Kurth, J., and Leister, D. 2002. Mode of amplification and reorganization of resistance genes during recent Arabidopsis thaliana evolution. Mol. Biol. Evol. 19:76-84.

Rose, L. E., Bittner-Eddy, P. D., Langley, C. H., Holub, E. B., Michelmore, R. W., and Beynon, J. L. 2004. The maintenance of extreme amino acid diversity at the disease resistance gene, RPP13, in Arabidopsis thaliana. Genetics 166:1517-1527.

Roy, S. W., and Gilbert, W. 2005. Rates of intron loss and gain: Implications for early eukaryotic evolution. Proc. Natl. Acad. Sci. U.S.A. 102, 5773-5778.

Sharp, P. A. 1981. Speculations on RNA splicing. Cell 23:643-646.

Smith, S. M., Pryor, A. J., and Hulbert, S. H. 2004. Allelic and haplotypic diversity at the $r p 1$ rust resistance locus of maize. Genetics. 167:1939-1947.

Thompson, J. D., Gibson, T. J., Plewniak, F., Jeanmougin, F., and Higgins, D. G. 1997. The ClustalX windows interface: Flexible strategies for multiple sequence alignment aided by quality analysis tools. Nucleic Acids Res. 25:4876-4882.

Wang, Z. X., Yano, M., Yamanouchi, U., Iwamoto, M., Monna, L., Hayasaka, H., Katayose, Y., and Sasaki, T. 1999. The Pib gene for rice blast resistance belongs to the nucleotide binding and leucine-rich repeat class of plant disease resistance genes. Plant J. 19:55-64.

Wei, F., Wing, R. A., and Wise, R. P. 2002. Genome dynamics and evolution of the Mla (powdery mildew) resistance locus in barley. Plant Cell 14:1903-1917.

Wu, T. C., and Lichten, M. 1994. Meiosis-induced double-strand break sites determined by yeast chromatin structure. Science 263:515-517.

Xu, X., Hsia, A. P., Zhang, L., Nikolau, B. J., and Schnable, P. S. 1995. Meiotic recombination break points resolve at high rates at the $5^{\prime}$ end of a maize coding sequence. Plant Cell 7:2151-2161.

Yang, Z. 1997. PAML: A program package for phylogenetic analysis by maximum likelihood. Comput. Appl. Biosci. 13:555-556.

Yang, Z., Nielsen, R., Goldman, N., and Pedersen, A. M. 2000. Codonsubstitution models for heterogeneous selection pressure at amino acid sites. Genetics 155:431-449.

Zhou, B., Chen, Z. H., Han, B., and Hong, G. F. 2001. Identification and structural analysis of a class of potentially transposable solo-LTR in rice genome. Acta bioch. Bioph. Sin. 33:158-162.

Zhou, B., Qu, S., Liu, G., Dolan, M., Sakai, H., Lu, G., Bellizzi, M., and Wang, G.-L. 2006. The eight amino acid differences within three leucine-rich repeats between Pi2 and Piz-t resistance proteins determine the resistance specificity to Magnaporthe grisea. Mol. Plant-Microbe Interact. 11: 1216-1228.

Zhang, J. 2003. Evolution by gene duplication:An update. Trends Ecol. Evol. 18:292-298.

\section{AUTHOR-RECOMMENDED INTERNET RESOURCES}

Arizona Genomics Institute website: www.genome.arizona.edu Bioinformatics Technology Network website: btn.genomics.org.cn The Institut Pasteur Matcher program: bioweb.pasteur.fr/seqanal/interfaces/matcher.html

Softberry website: www.softberry.com/berry.phtml

Texas A\&M University Laboratory for Plant Genomics and GENEfinder Genomic Resource website: hbz7.tamu.edu/index.htm 